\title{
Relationships between SHANK3 and Autism Spectrum Disorder
}

\author{
Zikuan Liu ${ }^{1, *}$ \\ ${ }^{1}$ US Center for Int'l Combined Curricula (UCICC).
}

\begin{abstract}
Autism Spectrum Disorder is a term that is used to describe people who have difficulties communicating with others and limited actions repetitive actions. Autism could be caused by both genetic and environmental conditions, and the Shank3 gene in the Shank family is one of the high-risk genes that has been found as one of the factors. However, although a lot of researches have been done to find out the relationships between Shank3 and autism, it is known by only a few people. This article will briefly talk about autism and Shank 3 and review how Shank 3 mutation causes autism and the changes in the brain and behaviour.
\end{abstract}

\section{Introduction}

Autism Spectrum Disorder (ASD), also called autism, is a disorder that very common in life, has a high probability of passing it on to offspring and in various forms. According to statistical researches done before, there are approximately 52 million people have autism around the world, which is around $0.76 \%$, and the frequency of having autism is found around 4 times higher in male than in female [1]. These people who have autism may have different degrees of social-communicative problems in different situations and keep doing something the same [2, 3]. Since ASD may seriously influence people's daily life, a huge number of researches have been done to try to cure this problem. It is found that autism could be caused by several genes. Some of these genes are Cntnap2, Neuroligins, Neurexins, and Shanks [4]. Among these genes, the Shanks gene family has been identified as one of the high-risk genes that will cause idiopathic Autism Spectrum Disorder [5].

$\mathrm{SH} 3$ and multiple ankyrin repeat domains 3 (SHANK3), also known as DEL22q13.3, PROSAP2, or PSAP2, is a member of the SHANK protein family. The other two family members are SHANK1 and SHANK2. All of them are encoded by corresponding genes (Shank3, Shank1, and Shank2). SHANK proteins are multidomain scaffold proteins located at excitatory synapses, playing an important role in connecting and securing other structures of the synapses. Although all the members in the SHANK protein family could be factors that cause Autism Spectrum Disorder and other related diseases, SHANK3 protein is considered as the most common and possible factor that causes autism by previous scholarships $[5,6]$.

Although both ASD and SHANK3 have been sufficient researched, the relationship between them has not been widely known by people. In this passage, we will review Autism Spectrum Disorder and SHANK3, then analysis the relationships between them through experiments taken before on mice, including the cause of

*Corresponding author:guanghua.ren@gecacademy.cn the disorder, the transform of the brain caused by the disorder, and the changes of the behaviour.

\section{Autism Spectrum Disorder}

Autism Spectrum Disorder is defined by the Diagnostic and Statistical Manual of Mental Disorders -5 th edition (DSM-5) in order to describe people who have lack social communication and interaction in various environments and have limited and repetitive ways of behaviour, interests, or activities. It is a general definition of disorders that include not only typical autistic disorder but also Asperger's disorder, childhood disintegrative disorder, and pervasive developmental disorder not otherwise specified (PDD-NOS) [3]. Statistics done by the Global Burden of Disease (GBD) and the World Health Organization (WHO) shows that the frequency of having autism is found to be higher in male than in female, which is about 2 to 5 times greater [1]. Moreover, about $18.7 \%$ of infants who have at least one older sibling have the disorder. The risk of getting autism for male infants is increased by 2.8 times, and it tripled if the infants have more than one older affected siblings [7]. According to the GBD study in 2010, there are approximately 52,000,000 people have autism around the world, which is $0.76 \%$. Evaluated by the Centers for Disease Control and Prevention (CDC), 1.68\% of children in the United States are considered with ASD $[1,2]$.

\subsection{Behaviour Feature}

People with autism are found to have remarkable features. Previous researches show that they may have an unusual social approach and failure to communicate with others, reducing sharing their interests and feelings, disable to lead or respond to social interactions; they may not be able to integrate verbal and nonverbal communication; they may also find it difficult to make friends. They keep doing what they used to do and express themselves by using 
fixed ways; they have fixed interests and focus on the interests in an unusual way; they may make high or no response to what happened near them or have an abnormal interest in the environment [2].

Furthermore, it is also found that compete with male, females with autism show greater symptoms in social communications and interactions, but fewer in restricted interests; they have lower ability overall, including verbal and non-verbal IQ and language processing; they have a lower adaptive function in social, communication and daily living skills; and they have greater problems in general and externalizing behaviour problems, and irritability and lethargy [8].

\subsection{Brain structure change caused by ASD}

Studies on infants with autism, which used structural neuroimaging, have shown that several brain structure changes may cause by autism. High-risk autistic infants have a higher level of extra-axial cerebrospinal fluid than normal and cause the first detectable behaviour change, lead to early motor deficits, but the brain size is normal; the growth rate of the cortical surface increased from 6 months to 12 months, lead to the over-growth of sensorimotor processing cortical area, and cause sensory and attention problems; and the growth rate of the total volume of the brain increased between 12 months and 24 months, caused specific social deficits [9].

Other studies also founded that specific brain structure change will cause autism, and several brain regions, including the Cerebellum, Amygdala, and Frontal cortex, are associated with autism.

Cerebellum. Autopsy analysis of individuals with autism shows that Purkinje neurons, which is the sole output of the cerebellum, decreased significantly in total numbers and density; further experiments taken on mouse support the deficits of Purkinje cell could cause autism.

Amygdala. Many researchers suggest that Amygdala is important in the neuropathology of autism. It is widely assumed that the amygdala will overgrow after birth but slow down or even become smaller in the end. Further researches are still needed to support the hypothesis.

Frontal cortex. Previous researches show that the stop developing until adolescence after the latter growth of the children [10].

\subsection{Genetic and environmental causes of ASD}

A lot of researches show that the cause of autism can be both genetic and environmental. There are over hundreds of genes related to autism, like Cntnap2, Neuroligins, Neurexins, and Shanks. Cntnap2 knock-out mice show reduced social interactions and increased repetitive behaviour, which is ASD-related behaviours. Neuroligin proteins are encoded by X-linked genes like NLGN4. NLGN4 knockout mice show reduced social interaction, ultrasonic vocalizations, and total volume of the brain. The SHANK family encodes scaffolding proteins. SHANK3, which could bind to neuroligins, suggests to has higher frequency and penetrance than SHANK1 and SHANK2. Most SHANK knock-out mice show the reduction of basal neurotransmission and abnormal neuron morphology [4].

Nevertheless, recent researches also show that more than $40 \%$ to $50 \%$ of autism could be caused by environmental factors. Some of the environmental factors, such as vaccination, maternal smoking, thiomersal exposure, and Assisted reproductive technology (ART), have been proved that they do not associate with the risk of having autism. In contrast, high-age parents, birth complications associated with trauma or ischemia and hypoxia, and other factors associated with pregnancy are highly related to autism. Some heavy metals may also cause autism [11].

\section{SHANK3 gene and protein}

\subsection{Gene position and expression}

The Sh3 and multiple ankyrin repeat domains 3 (Shank3) gene, also known as ProSAP2, is a protein coding gene in the Shank gene family. The Shank3 gene has been found located on human chromosome $22 \mathrm{q} 13.33$, which is on the end of the long arm of human chromosome 22, and has 23 exons (Fig.1A). Moreover, in the house mouse, the (a)

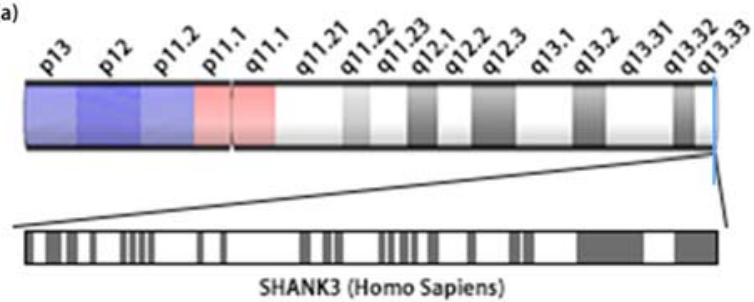

(b)

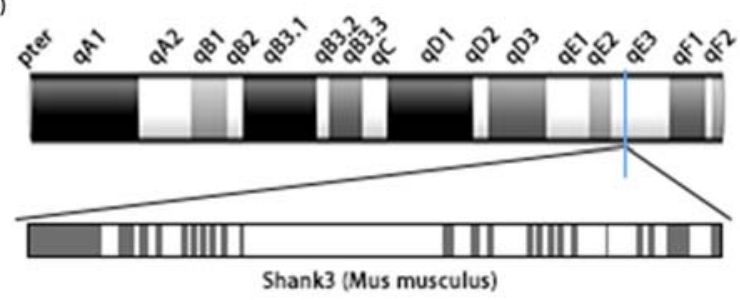

Fig. 1. Shank3 gene in human and mouse.

(a) Human Shank3 gene. (b) Mouse Shank3 gene. These two figures show the position of Shank3 gene and the position of exons on the gene (grey region).

total volume of brain and white and grey matter increased significantly in infants with autism by using MRI. The total weight of dorsolateral and medial prefrontal cortices is also found to increase in children with autism. Recently, another large research shows that the thickness of the cortex increased in the early stage and then decreased or
Shank3 gene is located on mouse chromosome $15 \mathrm{qE} 3$, which is on the middle of chromosome 15 , and has 24 exons (Fig.1B). Previous researchers have found the ribonucleic acid (RNA) of the gene is highly expressed in the human spleen, which reached 32.723 RPKM (reads per kilobase per million reads placed), fat, which reached 
31.723 RPKM, and brain, which reached 8.212 RPKM (Fig.2A). Furthermore, in mice, the RNA is highly expressed in adult lung, which is around 45.608 RPKM, adrenal, which is around 22.294 RPKM, and cerebellum, which is around 19.573 RPKM (Fig.2B).

\subsection{Protein structure and domain}

The SHANK3 protein coded by its gene is a multidomain scaffolding protein located in post-synapses. It has been considered as the possible candidate that causes autism or other neurotic disorders since it is suggested that SHANK3 proteins play a major role in the post-synaptic density (PSD), synapse formation and dendritic spine maturation, and functions just like a scaffold, connecting and sustaining other proteins, neurotransmitter receptors, and ion channels. The protein has several domains, including Shank/ProSAP N-terminal (SPN), Ankyrin
[12] ANK domain is a 33 aa protein domain, also on the $\mathrm{N}$-terminal part of SHANK3. It is one of the most abundant repeat motifs, which usually acts as a scaffold for interactions between proteins in progress such as cell cycle, transcriptional regulation, signal transduction, vesicular transport, and inflammatory response. It has been connected to several human diseases.[13] SH3 domain is a 52 aa protein domain. It plays a variety of roles on cells, including regulating enzymes, changing the subcellular localization of signaling pathway components, and mediating the formation of multi protein complex assemblies. [14] PDZ domain is a 90 aa protein domain. It exists in a variety of Eumetazoan signalling molecules. It may be responsible for specific protein-protein interactions since most PDZ domains bind to C-terminal

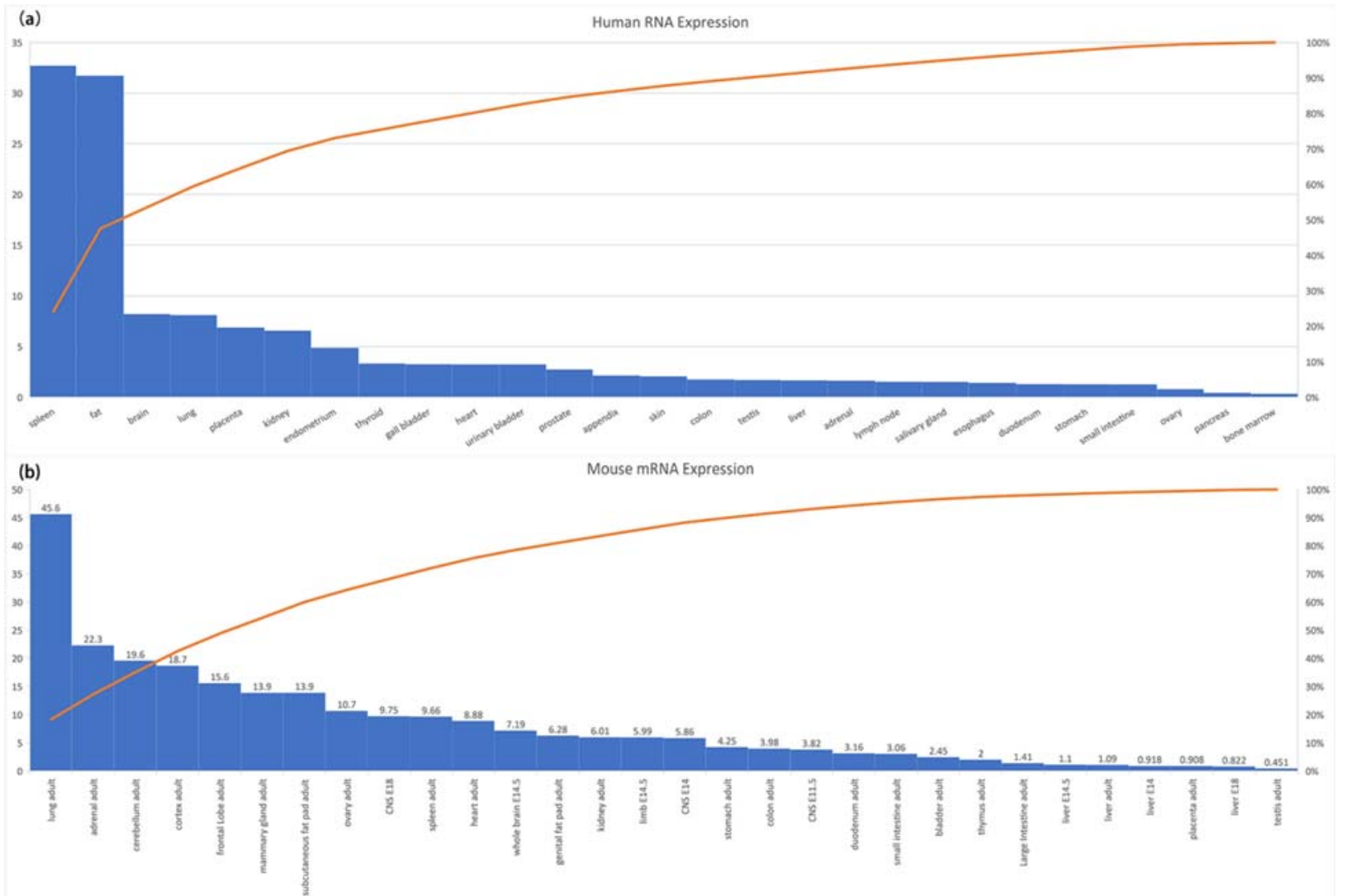

Fig. 2. RNA Expression in Human and mouse.

(a) HPA RNA-seq in human 27 different tissues. (b) Mouse RNA expression data from ENCODE project. The horizontal axis shows the name of the tissue. The left vertical axis shows the expression of Shank3 RNA in tissue in RPKM. The right vertical axis shows the proportion of each tissue's RNA expression to the whole. The line shows the cumulative percentage of the expression. The data comes from National Center of Biotechnology Information (NCBI).

repeat (ANK), Src Homology 3 (SH3), postsynaptic density protein 95 - discs large homologue 1 - zonula occludens 1/2 (PDZ), proline-rich region (PRO) and sterile alpha motif (SAM) (Fig.3). SPN domain is an 87 amino acid (aa) protein domain on the N-terminal part of Shank3. It has been found to have a similar structure as Four.1 protein, Ezrin, Radixin, Moesin, F0 subdomain (FERM_F0). Although its interactive proteins and the ways of affecting other proteins is still unclear, it has been proved that mutations in the domain could cause autism. 


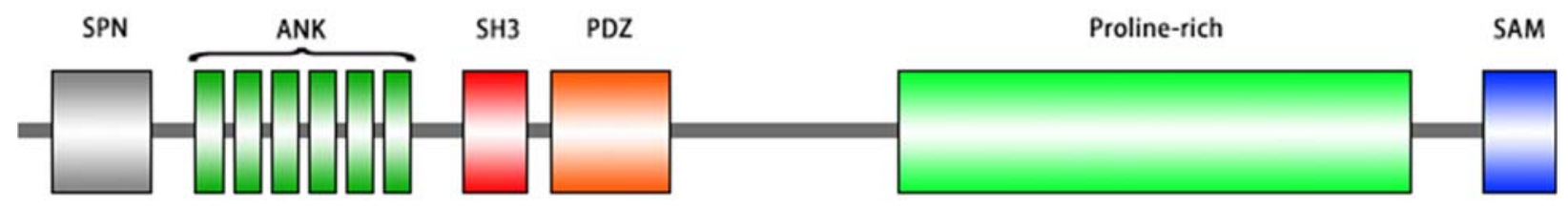

\section{SHANK3 Protein}

Fig. 3. SHANK3 protein domains.

peptides and have been shown to bind to internal peptides and even lipids.[15] PRO region is a region that may contain more than one protein domain and connect several other proteins.[16] SAM domain is a 66 aa protein domain. It is a protein-protein interaction domain. This domain of the SHANK family is easy to a homopolymer, and highly enriched in the postsynaptic density, and acts as scaffolds to organize the assembly of postsynaptic proteins. The SAM domain of Shank3 protein can form large helical fibres.[17]

\subsection{Functions in post-synapses}

The SHANK3 scaffolding protein is a protein located at post-synapse that connects other synapse structures and maintains the structures in the right place via its domains (Fig. 4). It has not been found that the SPN domain has any interactive structures, but it has been proved that mutations on this domain will influence the connection between SHANK3 domains and other structures. The ANK domain interacts with Sharprin, and F-actin via $\alpha$ - fodrin. The SH3 domain connects with GRIP, which interacts with AMPAR and EphR. AMPAR is an ion channel and EphR interacts with Ephrin in the presynapse. The PDZ domain connects with PSD-95 via SAPAP/GKAP, and PSD-95 binds NMDAR and NLGN. NMDAR is also an ion channel and NLGN interacts with NRXN, which located at the pre-synapse. The protein-rich region binds Homers and Cortactin. Homers connect mGluR and Cortactin binds F-actin. The SAM domain could bind other SHANK proteins. [18-20]

\section{Relationships between the two}

\subsection{ASD caused by SHANK3 mutation}

Researches have proved that mutations on SHANK3 will cause abnormal neurotic development and autism-like behaviours. Several recent experiments about mutations on SHANK3 and its isoforms are listed below (Table. 1). These researches show the exterior and neurotic

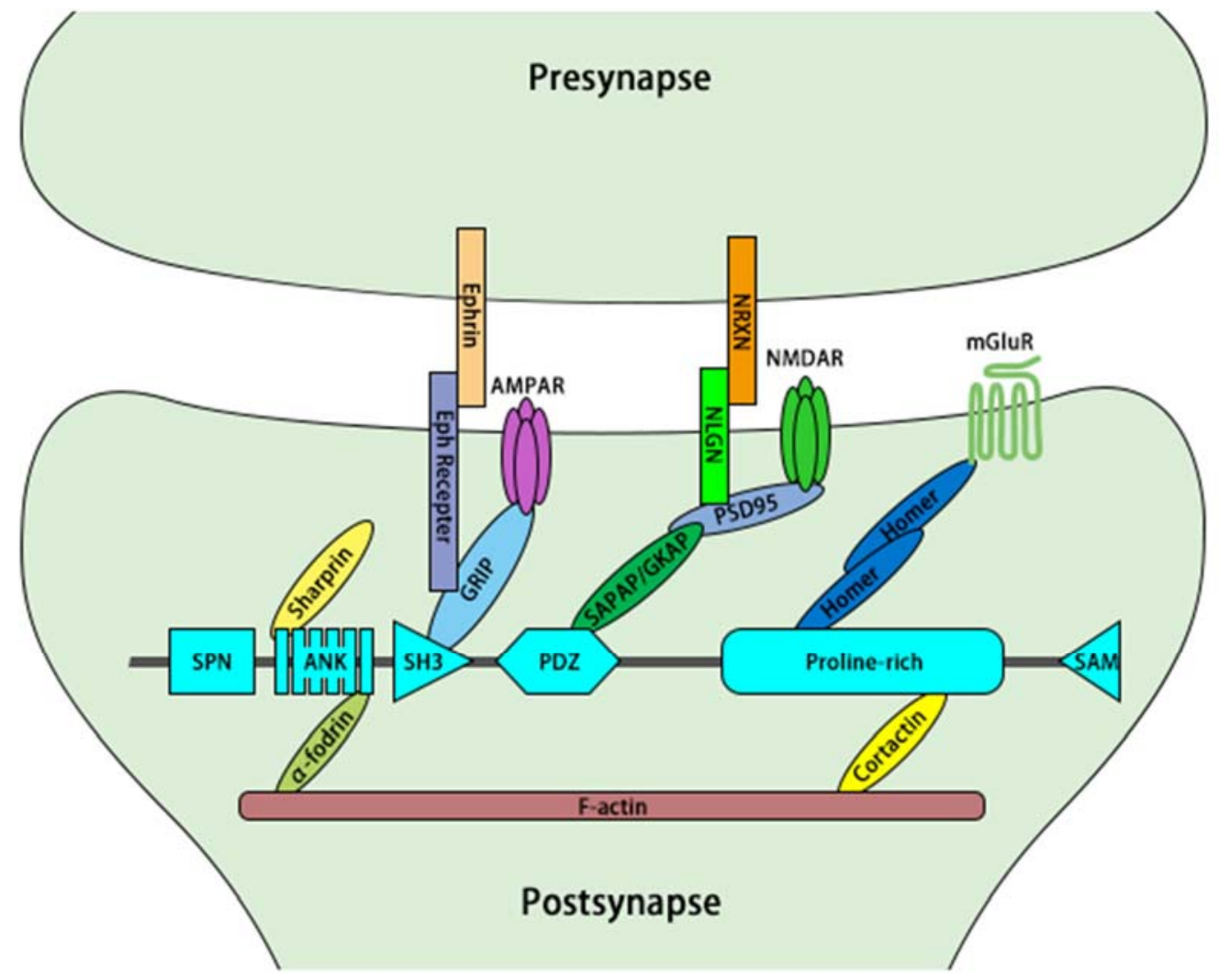

Fig .4. SHANK3 protein and associated structures in synapse. 
Table 1. Recent research on autism-related Shank3 mutations.

\begin{tabular}{|c|c|c|c|c|c|c|c|}
\hline Mutation & Organism & Organ & Exterior morphology & Neurotic morphology & Behaviour & Year & Refs \\
\hline $\begin{array}{l}\text { S685 knock- } \\
\text { in }\end{array}$ & mouse & $\begin{array}{lr}\begin{array}{l}\text { brain, } \\
\text { hippocampus }\end{array} & \begin{array}{r}\text { cortex, } \\
\text { striatum }\end{array} \\
\text { and }\end{array}$ & similar body weight & $\begin{array}{l}\text { reduce Shank3-ABI interaction; reduced } \\
\text { dendritic spines, impaired synaptic } \\
\text { transmission }\end{array}$ & $\begin{array}{l}\text { increased social dominance; increased allogrooming; altered } \\
\text { USV development }\end{array}$ & 2019 & [21] \\
\hline InsG3680 & mouse & $\begin{array}{l}\text { brain, cortex and } \\
\text { striatum }\end{array}$ & NA & changes in SNO-proteome & $\mathrm{NA}$ & 2018 & [22] \\
\hline $\begin{array}{l}\text { Shank3B } \\
\text { knock-out }\end{array}$ & mouse & brain, prefrontal & $\mathrm{NA}$ & $\begin{array}{l}\text { reduce prefrontal connection; reduced } \\
\text { prefrontal GM volume; reduced short- } \\
\text { range projection density }\end{array}$ & $\begin{array}{l}\text { unaltered total sniffing and social communication; a trend for } \\
\text { reduced sociability; reduced USV frequency }\end{array}$ & 2019 & [23] \\
\hline $\begin{array}{l}\text { Shank3B } \\
\text { knock-out }\end{array}$ & zebra fish & brain & $\begin{array}{l}\text { tail bending; reduced } \\
\text { melanin content in eye(in } \\
\text { early stage) }\end{array}$ & $\begin{array}{l}\text { delayed neurotic development; reduced } \\
\text { homer1 and synaptophysin protein levels }\end{array}$ & $\begin{array}{l}\text { impaired locomotor activity; abnormal repeated action; } \\
\text { unusual social interaction }\end{array}$ & 2018 & [25] \\
\hline $\begin{array}{l}\text { Shank3 exon } \\
12\end{array}$ & monkey & brain & NA & $\begin{array}{l}\text { reduced brain activity (lower glucose } \\
\text { metabolism) }\end{array}$ & $\begin{array}{l}\text { delayed development and vocalization; reduced social } \\
\text { communication; repetitive and anxiety-like behaviour }\end{array}$ & 2018 & [26] \\
\hline $\begin{array}{l}\text { Shank3 } \\
\text { knock-out }\end{array}$ & mouse & brain, whole & $\begin{array}{l}\text { no significant different } \\
\text { found in brain volume }\end{array}$ & NA & $\begin{array}{l}\text { reduced respose to pro-social } 50-\mathrm{kHz} \text { USV; normal response } \\
\text { calls to pro-social } 50-\mathrm{kHz} \text { USV, deficit in social interactions; } \\
\text { reduced USV in male pups }\end{array}$ & 2019 & [24] \\
\hline $\begin{array}{l}\text { Shank3 } \\
\text { knock-out }\end{array}$ & mouse & gastrointestine & $\begin{array}{l}\text { reduced villi length and } \\
\text { crypt depth; changed } \\
\text { microbiome }\end{array}$ & $\mathrm{NA}$ & $\mathrm{NA}$ & 2019 & [27] \\
\hline
\end{tabular}

morphology and behaviours of different SHANK3 mutant organisms. The mutation column exhibits the position of the mutation; the organism column displays the model organism used in the experiments; the organ column provides a list of main observation organs in the experiments.

\subsection{Neurotic and behavioural changes}

According to the table above, which shows the researches related to SHANK3 and autism done in recent 3 years, in most situation, mutations on Shank3 gene will cause abnormal neurotic morphology, such as reduced interactions and connections between synapses, lower synapses density and delayed or unproper neurotic development. The mutations will also cause unusual behaviour, for example, reduced social communications and repetitive and anxiety like behaviours. However, the outlook of the organism will not change too much in most situations.

S685 has been found as a phosphorylation site by $\mathrm{Li}$ Wang et al.[21] The site could influence the interaction between Shank3 and Abelson interactor (ABI), and mutation on $\mathrm{S} 685$ can reduce the interaction between them. By using the S685 mutant mouse models, it has found that the loss of the interaction could cause the alteration of social behaviours. The mouse models represent increased social dominance and allogrooming and slow-developed ultrasonic vocalization (USV). Alteration also shows in neurotic development, include reduced Shank3-ABI interaction and dendric spines, and impaired synaptic transmission. In contrast, the S685 knock-in mice have similar body weight, protein abundance and isotype specific expression patterns as the wild type (WT) mice.

The InsG3680 mice were reported by Haitham Amal et al.[22] The mice have a single guanine nucleotide $(\mathrm{G})$ insertion, which is associated with ASD patient, at position 3680 of the cDNA. The mutation leads to the change of the S-nitrosylation (SNO), and this may lead to alteration of synaptic functions.

Shank3B is one isoform of Shank3. The Shank3B knock-out mice experimented by Marco Pagani et al.[23] have been found a reduced prefrontal connection, reduced prefrontal grey matter volume and decreased short-range projection density. It has turned out that these alterations in neurotic system will cause several abnormal behaviours. Although the experiment found that the mice have unaltered total sniffing and social communication, they tend to reduce sociability and reduce USV frequency. Moreover, in experiments reported by Elizabeth L Berg et al.[24], the Shank3B knock-out mice represent reduced response to pro-social $50-\mathrm{kHz}$ USV, deficit in social interaction and reduced USV action in male pups. But the response calls to pro-social $50-\mathrm{kHz}$ USV shows normal, and there is no significant different found in brain volume between mutant mice and WT mice.

Furthermore, the Shank3B knock-out zebra fish, experimented by Chun-xue Liu et al.[25], shows impaired locomotor activity, abnormal repeated action and unusual social interaction in behaviour. It has also found that the Shank3B-null zebra fish have delayed neurotic development and reduced homer1 and synaptophysin protein levels. In early developmental stage, the tail of the fish also been found bending, and the melanin content in eye is reduced.

The mutation on Shank3 exon 12 also caused delayed development and vocalization, reduced social communication and repetitive and anxiety-like behaviour on monkey.[26] In neurotic morphology, it has been found that the mutant monkey has lower glucose metabolism than WT monkey in brain. This turns out that the brain activity is reduced in the mutant monkey.

Last but not the least, it is also been found that Shank3 knock-out mice could have gastrointestinal problems.[27] Experiments done by Ann Katrin Sauer et al. turns out that mutant mice have reduced villi length and crypt depth and changed microbiome.

\subsection{Treatments}

As the researches on Shank3 gene, SHANK3 protein and autism spectrum disorder go further, more ways that could treat the disease have been developed. Experimented by Zhuchi Tu,[26] Fluoxetine, a common medicine that used to treat major depression and OCD, could treat autism effectively. After 2 weeks' treatment, it turned out that the repetitive behaviour of the mutant monkey has greatly improved, and the time of social activities also increased. The mutant monkey increased the time of eye contact and 
the frequency of playing or staying alone decreased after receiving the treatment. Also, the brain glucose metabolism returned to normal. Moreover, Luye Qin et al.[28] found that Histone deacetylase (HDAC) inhibitor romidepsin could also be used to treat autism. According to the experiment, by giving the mutant mice low dose of romidepsin, the social deficit of mutant mice could be treated persistently. The NMDAR function and F-actin could also be recovered.

\section{Conclusion}

Autism Spectrum Disorder is a kind of disorder that very common, highly inheritable, and complex. Shank3, as a high-risk gene that may cause autism, has been researched for a long time. In this review, we talked about autism, Shank3, and the relationships between them. However, although we have already known that mutations in Shank3 gene will cause autism-like behaviour and abnormal, neurotic development, the actual way that the mutation causes the phenomenon and the function of each domain in SHANK3 protein have not been fully discovered.

\section{References}

1. C. Lord, T. S. Brugha, et al., Autism spectrum disorder, 6, 1-23.(2020)

2. H. Hodges, C. Fealko, and N. Soares, AME Publishing Company, Autism spectrum disorder: definition, epidemiology, causes, and clinical evaluation, in Translational pediatrics, 9, S55S65.(2020)

3. A. P. Association, American Psychiatric Pub, Diagnostic and statistical manual of mental disorders (DSM-5®).(2013)

4. T. M. Kazdoba, P. T. Leach, and J. N. Crawley, Behavioral phenotypes of genetic mouse models of autism, in Genes, brain, and behavior, 15, 7-26.(2016)

5. Y. H. Jiang and M. D. Ehlers, Modeling autism by SHANK gene mutations in mice, in Neuron, $\mathbf{7 8}, 8-$ 27.(2013)

6. P. Monteiro and G. Feng, SHANK proteins: roles at the synapse and in autism spectrum disorder, in Nat Rev Neurosci, 18, 147-157.(2017)

7. S. Ozonoff, G. S. Young, et al., American Academy of Pediatrics, Recurrence risk for autism spectrum disorders: a Baby Siblings Research Consortium study, in Pediatrics, 128, e488-e495.(2011)

8. T. W. Frazier, S. Georgiades, et al., Behavioral and cognitive characteristics of females and males with autism in the Simons Simplex Collection, in Journal of the American Academy of Child and Adolescent Psychiatry, 53, 329-40.e403.(2014)

9. M. D. Shen and J. Piven, Les Laboratoires Servier, Brain and behavior development in autism from birth through infancy, in Dialogues in clinical neuroscience, 19, 325-333.(2017)

10. A. P. A. Donovan and M. A. Basson, John Wiley and
Sons Inc., The neuroanatomy of autism - a developmental perspective, in Journal of anatomy, 230, 4-15.(2017)

11. A. Modabbernia, E. Velthorst, and A. Reichenberg, BioMed Central, Environmental risk factors for autism: an evidence-based review of systematic reviews and meta-analyses, in Molecular autism, $\mathbf{8}$, 13-13.(2017)

12. F. Hassani Nia, D. Woike, et al., BioMed Central, Targeting of $\delta$-catenin to postsynaptic sites through interaction with the Shank3 N-terminus, in Molecular autism, 11, 85-85.(2020)

13. Q. Cai, T. Hosokawa, et al., Shank3 Binds to and Stabilizes the Active Form of Rapl and HRas GTPases via Its NTD-ANK Tandem with Distinct Mechanisms, in Structure, 28, 290-300.e4.(2020)

14. A. Musacchio, M. Saraste, and M. Wilmanns, Highresolution crystal structures of tyrosine kinase $\mathrm{SH} 3$ domains complexed with proline-rich peptides, in Nat Struct Biol, 1, 546-51.(1994)

15. S. K. Ponna, S. Ruskamo, et al., Structural basis for PDZ domain interactions in the post-synaptic density scaffolding protein Shank3, in $J$ Neurochem, 145, 449-463.(2018)

16. T. Sarowar and A. M. Grabrucker, Hindawi Publishing Corporation, Actin-Dependent Alterations of Dendritic Spine Morphology in Shankopathies, in Neural plasticity, 2016, 8051861-8051861.(2016)

17. M. J. Knight, M. K. Joubert, et al., Zinc binding drives sheet formation by the SAM domain of diacylglycerol kinase $\delta$, in Biochemistry, 49, 96679676.(2010)

18. A. Grabrucker, A Role for Synaptic Zinc in ProSAP/Shank PSD Scaffold Malformation in Autism Spectrum Disorders, in Developmental neurobiology, 74.(2014)

19. J. Costales and A. Kolevzon, Phelan-McDermid Syndrome and SHANK3: Implications for Treatment, in Neurotherapeutics : the journal of the American Society for Experimental NeuroTherapeutics, 12.(2015)

20. Y. Vyas and J. Montgomery, The role of postsynaptic density proteins in neural degeneration and regeneration, 11, 906-907.(2016)

21. L. Wang, K. Pang, et al., An autism-linked missense mutation in SHANK3 reveals the modularity of Shank3 function, in Molecular psychiatry, 25, 25342555.(2020)

22. H. Amal, B. Barak, et al., Shank3 mutation in a mouse model of autism leads to changes in the S-nitrosoproteome and affects key proteins involved in vesicle release and synaptic function, in Molecular psychiatry, 25, 1835-1848.(2020)

23. M. Pagani, A. Bertero, et al., Society for Neuroscience, Deletion of Autism Risk Gene Shank3 Disrupts Prefrontal Connectivity, in The Journal of neuroscience : the official journal of the Society for Neuroscience, 39, 5299-5310.(2019) 
24. E. L. Berg, N. A. Copping, et al., Developmental social communication deficits in the Shank3 rat model of phelan-mcdermid syndrome and autism spectrum disorder, in Autism research : official journal of the International Society for Autism Research, 11, 587-601.(2018)

25. C.-X. Liu, C.-Y. Li, et al., BioMed Central, CRISPR/Cas9-induced shank3b mutant zebrafish display autism-like behaviors, in Molecular autism, 9, 23-23.(2018)

26. Z. Tu, H. Zhao, et al., Oxford University Press, CRISPR/Cas9-mediated disruption of SHANK3 in monkey leads to drug-treatable autism-like symptoms, in Human molecular genetics, 28, 561-571.(2019)

27. A. K. Sauer, J. Bockmann, et al., MDPI, Altered Intestinal Morphology and Microbiota Composition in the Autism Spectrum Disorders Associated SHANK3 Mouse Model, in International journal of molecular sciences, 20, 2134.(2019)

28. L. Qin, K. Ma, et al., Social deficits in Shank3deficient mouse models of autism are rescued by histone deacetylase (HDAC) inhibition, in Nature neuroscience, 21, 564-575.(2018) 